\title{
Laboratory Diagnosis of Congenital Syphilis by Immunoglobulin M (IgM) and IgA Immunoblotting
}

\author{
JOHN L. SCHMITZ, ${ }^{1}$ KARLA S. GERTIS, ${ }^{1}$ CHARLES MAUNEY, ${ }^{2}$ LOLA V. STAMM, ${ }^{3}$ \\ AND JAMES D. FOLDS ${ }^{1 *}$ \\ Clinical Microbiology/Immunology Laboratories, University of North Carolina Hospitals, ${ }^{1}$ and Department of \\ Epidemiology, School of Public Health, University of North Carolina, ${ }^{3}$ Chapel Hill, North Carolina 27514, \\ and Carolinas Medical Center, Charlotte, North Carolina $28232^{2}$
}

Received 4 August 1993/Returned for modification 13 September 1993/Accepted 23 September 1993

\begin{abstract}
We screened cord blood or serum samples from 101 infants at risk for congenital syphilis and serum samples from their mothers for immunoglobulin G (IgG), IgM, and IgA antibodies to Treponema pallidum by Western blotting (immunoblotting). Clinical evaluation showed that six infants had signs and/or symptoms consistent with congenital syphilis. The sera from five of these infants were IgM blot positive, and four were IgA blot positive. Four asymptomatic infants had serologic evidence of congenital syphilis. The sera from three of these infants were IgM blot positive, and two were IgA blot positive. However, the IgM reactivity of the serum from one asymptomatic infant, which was also IgA positive, was abolished by protein G treatment. An IgM capture enzyme-linked immunosorbent assay corroborated the presence of IgM antibodies in six of seven IgM blot-reactive sera. Overall, for detection of symptomatic congenital syphilis, a sensitivity of $83 \%$ for IgM blotting and $67 \%$ for IgA blotting was obtained. The significance of positive IgM or IgA Western blots for asymptomatic infants requires further study to confirm infection in these infants.
\end{abstract}

As a consequence of the increased incidence of syphilis in women of childbearing age (6), cases of congenital syphilis have been on the rise in the United States (2). Although the early increase in 1990 was at least partly due to changes in the case definition of congenital syphilis (5), the continued increases reflect a true increase in disease incidence. The diagnosis of symptomatic congenital syphilis can be made on clinical grounds when the infection and treatment history of the mother is known. However, up to $60 \%$ of infants with congenital syphilis are asymptomatic at birth (15). Additionally, since the maternal treatment history is not always known, a reliable laboratory test to diagnose infection is desirable. For these reasons, there is great interest in improving methods of laboratory diagnosis of this disease.

Because standard serologic tests for syphilis detect both immunoglobulin (Ig) $\mathrm{G}$ and IgM, including transplacentally acquired maternal IgG, they cannot be used to provide a laboratory diagnosis of congenital syphilis when a single serum sample is tested (11). An exception to this occurs, however, when the titer for the infant is fourfold or more higher than that for the mother. A variety of IgM-specific assays have been developed to overcome this problem. The previously unreliable fluorescent treponemal antibody absorption (FTA-ABS) test for IgM (12) has been modified (19S IgM FTA-ABS assay) to improve its performance but is of limited availability (15). IgM-specific enzyme-linked immunosorbent assays (ELISAs) and Western blot (WB) (immunoblotting) tests for congenital syphilis have also been developed $(3,4,7,8,16,17,19,20$, $23-26,28,29,32)$. In addition to serologic tests, the rabbit infectivity test and PCR have been studied for their applicability to diagnosis of this disease and show promise $(9,24)$.

We evaluated the IgM and IgA WB as a screening test for the diagnosis of congenital syphilis in a group of 101 newborn

\footnotetext{
* Corresponding author. Mailing address: Clinical Microbiology/ Immunology Laboratories, Rm. 1035 PST, UNC Hospitals, Chapel Hill, NC 27514. Phone: (919) 966-5091. Fax: (919) 966-4526.
}

infants at risk for congenital syphilis. The IgM WB demonstrated good sensitivity for detection of symptomatically infected infants. However, its use for asymptomatically infected infants at risk for congenital syphilis requires further confirmation of infection by alternative means.

\section{MATERIALS AND METHODS}

Study population. Consecutive cord blood and serum samples from 101 infants at risk for congenital syphilis and serum samples from their mothers were collected over a 1-year period (February 1991 to February 1992) at the Carolinas Medical Center, Charlotte, N.C., and at the University of North Carolina Hospitals, Chapel Hill, N.C. An infant was defined as being at risk for congenital syphilis if it was born to a mother with a reactive serologic test for syphilis regardless of maternal treatment history. All maternal and infant samples were collected within $24 \mathrm{~h}$ of delivery except for one mother-infant pair, which was collected 2.5 months after delivery. Sera were stored frozen at $-20^{\circ} \mathrm{C}$ and not thawed until assayed by WB. For this retrospective study, WBs were performed blindly, without knowledge of the clinical findings for the infants.

Serologic tests for syphilis. All maternal and infant sera were tested for nontreponemal and treponemal antibodies by standard serologic tests. The Venereal Disease Research Laboratory (VDRL) test was performed as recommended by the manufacturer on all infant and maternal samples. Eighty-five of the maternal sera were also tested by the FTA-ABS test (Incstar Corporation, Stillwater, Minn.), performed according to the manufacturer's instructions.

Treponema pallidum preparation. $T$. pallidum Nichols was obtained by intratesticular inoculation of New Zealand White rabbits. When peak orchitis was reached, testicles were removed, rinsed in phosphate-buffered saline (PBS), and minced. PBS with $10 \%$ heat-inactivated normal rabbit serum was added to the minced testicles, and the treponemes were extracted by repeated rounds of agitation $(98 \mathrm{rpm})$ at $32^{\circ} \mathrm{C}$ followed by centrifugation at $400 \times g$ and aspiration of the 
supernatant. Supernatants were pooled and centrifuged at $10,000 \times g$ for $15 \mathrm{~min}$ at $4^{\circ} \mathrm{C}$. The treponemal cell pellet was resuspended in PBS, and the treponemes were quantitated by dark-field microscopy, aliquoted, and frozen at $-70^{\circ} \mathrm{C}$.

WB. $T$. pallidum organisms $\left(1.25 \times 10^{9}\right)$ were solubilized in $10 \mathrm{mM}$ Tris-1 mM EDTA-1\% sodium dodecyl sulfate (SDS) by boiling for $3 \mathrm{~min}$. Samples were then mixed with equal volumes of $2 \times$ sample buffer $(125 \mathrm{mM}$ Tris, $4 \%$ SDS, $20 \%$ glycerol, $2 \%$ 2-mercaptoethanol, $0.001 \%$ bromphenol blue). Proteins were separated by SDS-polyacrylamide gel electrophoresis (PAGE) in gels (14 by $16 \mathrm{~cm} ; 4 \%$ acrylamide stacking gels and $15 \%$ acrylamide separating gels) with $25 \mathrm{mM}$ Tris-192 $\mathrm{mM}$ glycine- $0.1 \%$ SDS running buffer (13). Gels were run at $150 \mathrm{~V}$ for $4 \mathrm{~h}$ (SE 400 Electrophoresis Unit; Hoefer Scientific, San Francisco, Calif.). An single lane of the gel contained molecular mass markers (SDS-PAGE standards, low range; Bio-Rad Laboratories Inc., Hercules, Calif.). Proteins were transferred to nitrocellulose $(0.45 \mu \mathrm{m})$ by the method of Towbin et al. (30) at $195 \mathrm{~mA}$ for $1 \mathrm{~h}$ and then at $50 \mathrm{~mA}$ for 2 h (Bio-Rad Trans-blot Cell; Bio-Rad Laboratories Inc.). Nitrocellulose sheets were blocked in PBS ( $7 \mathrm{mM} \mathrm{Na}_{2} \mathrm{HPO}_{4}, 2.8$ $\mathrm{mM} \mathrm{NaH} \mathrm{PO}_{4}, 145 \mathrm{mM} \mathrm{NaCl}$ [pH 7.2]) with $0.05 \%$ Tween 20 and $3 \%$ nonfat dry milk for $1 \mathrm{~h}$ at room temperature, rinsed in distilled water, cut into strips, and stored at $-20^{\circ} \mathrm{C}$ until use. Sera and controls were diluted 1:100 in PBS-Tween-milk and incubated with strips overnight at room temperature with agitation. After three washes in PBS-Tween-milk, appropriately diluted, isotype-specific, horseradish peroxidase-conjugated secondary antibodies to human IgG, IgM, or IgA (Organon Teknika Corporation, West Chester, Pa.) were added and incubated for $1 \mathrm{~h}$ at room temperature with agitation. The strips were washed three times, and the substrate 4-chloro-1-naphthol (Sigma Chemical Co., St. Louis, Mo.) was added and incubated at room temperature. Color development was stopped by rinsing the strips in distilled water. IgM and IgA blots were considered positive if any reactivity was apparent.

IgM capture ELISA. Cord blood or serum samples from 8 of 10 infants with serologic and/or clinical evidence of congenital syphilis were analyzed by an IgM capture enzyme-linked immunosorbent assay (ELISA; DCL-Captia Syphilis-M; Diagnostic Chemicals, Limited, Oxford, Conn.). The assays were performed according to the manufacturer's instructions.

IgM separation. The IgM fraction of cord blood or serum was obtained by protein $G$ affinity separation with Quik-Sep IgM vials (Isolab, Inc., Akron, Ohio). These vials contain recombinant protein $\mathrm{G}$, which removes all subclasses of $\mathrm{IgG}$ from serum. Briefly, $50 \mu$ l of serum was added to an absorption vial containing recombinant protein $\mathrm{G}$, vortexed for $10 \mathrm{~s}$, and placed on a rocking platform for $10 \mathrm{~min}$ at room temperature. A porous disk was then placed into the vial and pushed to the bottom, and the supernatant was removed and used for blots. The supernatant, which is collected at a 1:8 dilution, was diluted 1:12.5 (final dilution, 1:100), equivalent to the dilution of whole serum used. All samples from infants with clinical and/or serologic evidence of congenital syphilis were assayed with absorbed cord blood or serum in addition to whole cord blood and serum.

RF detection. Cord blood and serum samples from 8 of 10 infants with clinical and/or serologic evidence of congenital syphilis (Table 1) were tested for the presence of rheumatoid factor (RF) by latex agglutination (Rheuma-Lex; Trinity Laboratories Inc., Raleigh, N.C.). Assays were performed according to the manufacturer's instructions. Briefly, $40 \mu \mathrm{l}$ of serum was added to 1 drop of latex reagent, mixed, and rotated at 100 rpm for $2 \mathrm{~min}$. Agglutination reactions were observed under a
TABLE 1. Clinical and serologic findings for infants with suspected congenital syphilis ${ }^{a}$

\begin{tabular}{|c|c|c|c|c|c|c|}
\hline \multirow{2}{*}{$\begin{array}{c}\text { Infant } \\
\text { no. }\end{array}$} & \multicolumn{2}{|c|}{$\underset{\text { titer }}{\text { Serum VDRL }}$} & \multicolumn{2}{|c|}{$\mathrm{WB}^{\prime \prime}$} & \multirow{2}{*}{$\begin{array}{c}\text { IgM } \\
\text { ELISA }\end{array}$} & \multirow{2}{*}{$\mathrm{RF}$} \\
\hline & Mother & Infant & $\operatorname{IgM}$ & IgA & & \\
\hline \multicolumn{7}{|c|}{ Symptomatic } \\
\hline 21 & $1: 256$ & $1: 128$ & + & + & + & + \\
\hline 32 & $1: 64$ & $1: 512$ & + & + & + & + \\
\hline 50 & $1: 128$ & $1: 64$ & + & + & + & + \\
\hline 56 & $1: 64$ & $1: 16$ & - & - & + & - \\
\hline 66 & $1: 128$ & $1: 16$ & + & - & + & - \\
\hline 117 & 1:512 & $1: 256$ & + & + & $\mathrm{ND}^{c}$ & ND \\
\hline \multicolumn{7}{|c|}{ Asymptomatic } \\
\hline 68 & $1: 32$ & $1: 16$ & + & - & + & - \\
\hline 76 & $1: 256$ & $1: 128$ & + & - & - & ND \\
\hline 96 & $1: 32$ & $1: 2$ & - & + & ND & - \\
\hline 103 & $1: 2$ & $1: 2$ & $+^{d}$ & + & + & + \\
\hline
\end{tabular}

"Includes all infants with clinical and/or serologic (reactive IgM and/or IgA WB) evidence of congenital syphilis. Sera were not numbered consecutively for this study.

' + , reactivity to any protein; -, complete lack of reactivity.

"ND, not determined.

"IgM reactivity removed by absorption with protein $\mathrm{G}$.

bright light and scored as positive (any degree of agglutination) or negative (complete lack of agglutination).

Chart reviews. The medical records of all 101 infants at risk for congenital syphilis were examined retrospectively to determine whether any had signs or symptoms consistent with congenital syphilis at birth (11). When documented, the treatment history of the mother for syphilis was noted.

\section{RESULTS}

Serologic tests for syphilis in infants and their mothers. One hundred and one infants and 85 of the VDRL-reactive mothers were included in this study. Maternal VDRL titers ranged from reactive undiluted to 1:512. Eighty-one of the maternal sera were reactive by FTA-ABS (data not shown). VDRL titers for the infants ranged from nonreactive to 1:512. In only one case did an infant (no. 32) have a VDRL titer (1:512) higher than that of its mother (1:64, Table 1). This infant's sample was also reactive in IgM and IgA WB (discussed below).

Clinical findings for infants at risk for congenital syphilis. Analysis of the infants' medical records revealed six infants (no. $21,32,50,56,66$, and 117) with one or more clinical findings consistent with congenital syphilis (Table 1). The most frequent finding was evidence of bone involvement by X-ray (five infants), followed by prematurity (three infants), liver involvement (three infants), seizures (one infant), pneumonia (one infant), thrombocytopenia (one infant), hearing loss (one infant), and rash (one infant). A seventh infant (no. 76) without clinical signs had a reactive (1:1) cerebrospinal fluid VDRL test.

IgG WBs. Eighty-five of 101 maternal and all 101 infant cord blood and serum samples were analyzed by IgG WB. The WBs for six symptomatic infants (Table 1) and their mothers were compared (Fig. 1). In general, mother and infant IgG results were similar. However, sera from three infants (data for one not shown) with symptoms suggestive of congenital syphilis demonstrated a band of greater intensity than the mother's serum (Fig. 1, lanes 4 and 6). This band corresponded to a protein with a molecular mass of approximately $83 \mathrm{kDa}$. The pattern of IgG reactivity in asymptomatic infants with IgM- 


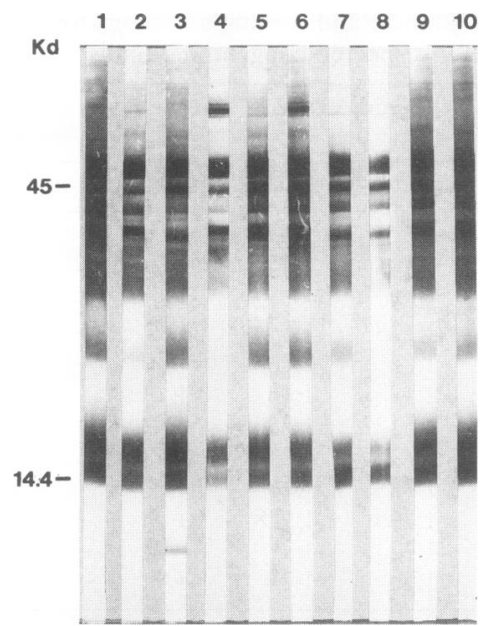

FIG. 1. IgG WB of maternal (odd-numbered lanes) and corresponding infant (even-numbered lanes) sera. All five infants had symptoms consistent with congenital syphilis. Lanes 1 and 2, mother and infant 21; lanes 3 and 4, mother and infant 32; lanes 5 and 6 , mother and infant 50; lanes 7 and 8, mother and infant 56; lanes 9 and 10 , mother and infant 66.

and/or IgA-reactive blots (Table 1) and their mothers was similar.

IgM WBs. All infant cord blood and serum samples and 85 of 101 maternal serum samples were analyzed for the presence of IgM antibodies to $T$. pallidum by WB with an IgM-specific secondary antibody. The effect of RF and IgG on IgM WB reactivity was assessed by absorption of $\mathrm{IgG}$ from the 10 infant samples listed in Table 1 with protein G. Eight of 101 infant sera demonstrated reactivity in IgM WBs (Table 1, Fig. 2). Five of the eight were from symptomatic infants, and the other three were from asymptomatic infants. Seven of the eight sera reacted with the $47-\mathrm{kDa}$ antigen. Variable reactivity was observed to antigens of $45 \mathrm{kDa}$ and lower. One serum (infant no. 76) reacted solely to an antigen of approximately $93 \mathrm{kDa}$ (not shown).

Absorption of IgG from infant cord blood and serum

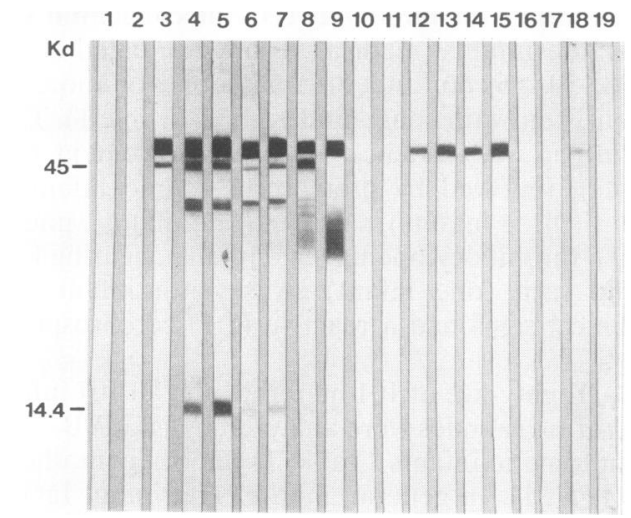

FIG. 2. Examples of reactive and nonreactive IgM WBs. Lane 1, negative control; lane 2, negative control absorbed with protein $\mathrm{G}$; lane 3, positive control. Lanes 4, 6, 8, 10, 12,14, 16, and 18, unabsorbed serum from infants $21,32,50,56,66,68,96$, and 103 , respectively. Lanes $5,7,9,11,13,15,17$, and 19, protein G-absorbed serum from infants $21,32,50,56,66,68,96$, and 103 , respectively.

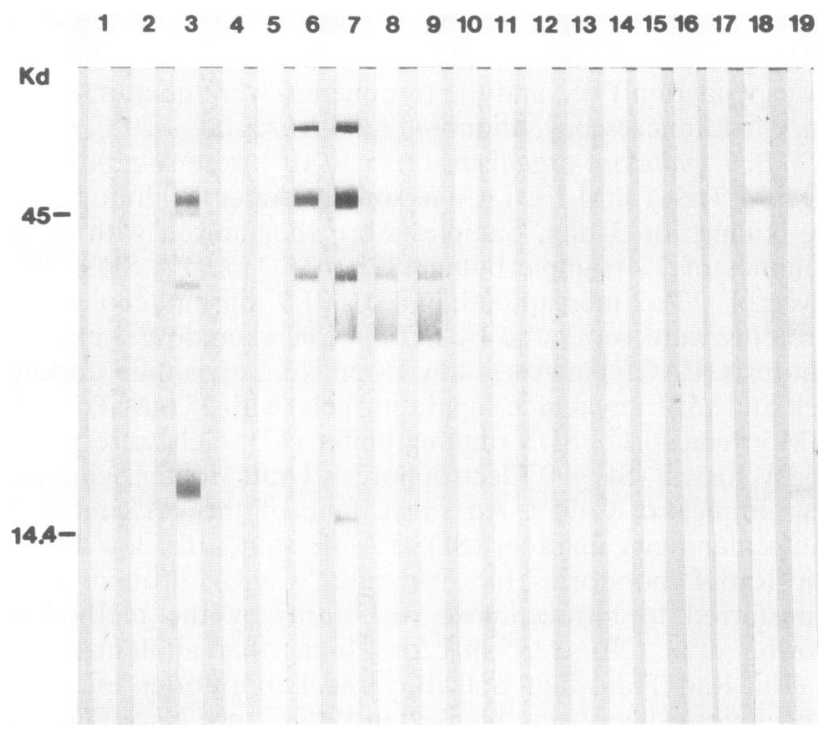

FIG. 3. Examples of reactive and nonreactive IgA WBs. Lane 1, negative control; lane 2, negative control absorbed with protein $\mathrm{G}$; lane 3, positive control. Lanes $4,6,8,10,12,14,16$, and 18, unabsorbed serum from infants $21,32,50,56,66,68,96$, and 103 , respectively. Lanes 5, 7, 9, 11, 13, 15, 17, and 19, protein G-absorbed serum from infants $21,32,50,56,66,68,96$, and 103 , respectively. The blot for infant 96 (lanes 16 and 17) gave a weak reaction to the $47-\mathrm{kDa}$ antigen which does not show up well on the photograph. The same weak reaction was obtained in two separate experiments.

samples with recombinant protein $\mathrm{G}$ both ablated reactivity to some proteins and enhanced reactivity to others. Infant sample 103 lost reactivity to the $47-\mathrm{kDa}$ band after absorption (Fig. 2, lane 19), while infant sample 50 lost reactivity to the $45-\mathrm{kDa}$ antigen but had increased reactivity to lower-molecular-mass antigens after absorption (Fig. 2, lane 9). The intensity of the 47-kDa band increased after absorption of sera 66 and 68 (Fig. 2 , lanes 13 and 15 ), as did the intensity of the $15-\mathrm{kDa}$ band with serum 21 (Fig. 2, lane 5).

IgA WBs. Since IgA antibodies are also markers of congenital infection, we analyzed infant sera by WB with an IgAspecific secondary antibody. Samples from the 10 infants listed in Table 1 were also analyzed after absorption with protein $\mathrm{G}$. Six of 101 infant serum samples demonstrated reactivity in the IgA WB (Table 1, Fig. 3). Four of the six were from symptomatic infants, and two were from asymptomatic infants. All six sera reacted to the $47-\mathrm{kDa}$ antigen, with variable reactivity to higher- and lower-molecular-mass antigens. Absorption of sera with recombinant protein $G$ did not abolish reactivity to any proteins but did increase the intensity of bands for several sera (Fig. 3, lanes 7, 9, and 19).

Confirmation of IgM WB reactivity by IgM capture ELISA. Eight of the 10 cord blood and serum samples from infants with clinical and/or serologic evidence of congenital syphilis were tested by IgM capture ELISA to corroborate the IgM WB findings (Table 1). Five of five sera $(100 \%)$ tested from the symptomatic infants gave positive IgM capture ELISA results, corroborating four of our IgM blot findings. Infant sample 56, which was negative by IgM WB, was positive by ELISA. There was not enough of the sixth serum for ELISA testing. Two of three samples from the asymptomatic group were positive by the IgM capture ELISA. Sample 103, which was negative by WB after absorption with protein $G$, was reactive in the ELISA. 
Correlation of clinical findings with IgM and IgA WB results. Of the six symptomatic infants identified in our study, five had reactive IgM blots (Table 1) and four had reactive IgA blots, yielding a sensitivity of 83 and $67 \%$, respectively. Four asymptomatic infants had serologic evidence of infection: no. 68 and 76 had positive IgM WBs, and no. 96 and 103 had positive IgA WBs (Table 1). One of the asymptomatic infants (infant 76) had a reactive cerebrospinal fluid VDRL test (Table 1). Combined use of IgM and IgA WBs yielded serologic evidence of infection for all four of these infants, whereas the use of either alone detected only two of the four infants. The IgM capture ELISA was positive for two of the three samples tested (Table 1).

As stated above, four mothers had false-positive screening tests (i.e., negative FTA-ABS results). These sera and their respective infant cord blood and serum samples were also tested by WB. Two of the maternal sera gave bands on the IgG WB, but none of the infant sera had any reactivity in an IgG, IgM, or IgA WB.

\section{DISCUSSION}

The diagnosis of congenital syphilis is often made clinically because standard serologic tests for syphilis, although useful in documenting maternal infection, do not distinguish maternal from infant antibody. In addition, many congenitally infected infants are asymptomatic at birth, and several months may pass before an increasing VDRL titer or persistent FTA-ABS reactivity can be documented to confirm congenital infection. Therefore, a reliable test to document congenital infection soon after birth would be very helpful in the management of infants at risk for congenital syphilis.

Serologic tests detecting IgM antibodies to infectious agents are useful in diagnosing congenital infections (1). Of the various techniques available to detect $T$. pallidum-specific IgM antibodies, such as 19S IgM FTA-ABS, IgM ELISA, and IgM WB, we chose the latter two to screen cord blood or serum from 101 infants born to mothers with positive screening tests for syphilis. WB has been shown to be a sensitive assay for the detection of antibodies to other infectious agents $(10,27)$. In addition, the documentation of reactivity to various proteins of the infectious agent provides a high degree of specificity. These attributes made WB an attractive choice for a serologic test for diagnosis of congenital syphilis.

Consistent with previous reports $(7,17,23,24,26,32)$, the IgM WB was very effective in confirming congenital syphilis in symptomatic infants. Overall, six infants in our study had symptomatic congenital syphilis. Five of these infants had IgM-reactive blots. The IgM WB-negative, symptomatic infant (no. 56) was born to a mother with evidence of a primary lesion, and therefore, low levels of IgM were probably present in this sample. Further optimization of the WB protocol may help to increase its sensitivity. The use of a tertiary antibody probe or an alkaline phosphatase-labeled secondary antibody might also increase the sensitivity of our WB system. In addition to symptomatic infants, IgM antibodies to $T$. pallidum were also present in two asymptomatic infants (no. 68 and 76). The mother of infant 68 was treated 2 months before delivery, and therefore we may have detected residual IgM antibody in a successfully treated infant. Infant 76's mother was previously treated but had a VDRL titer of 1:256 at delivery and 1:512 4 days before delivery, suggesting inadequate treatment or reinfection. Because of this high-titer maternal VDRL test and the infant's reactive cerebrospinal fluid VDRL test, we believe that the infant was asymptomatically infected.

Analysis of the sera from symptomatic infants by IgM capture ELISA corroborated our IgM blot findings. Five of six sera were positive by this method. Too little of the sixth serum was available for ELISA testing. The IgM WB-negative serum from infant 56 was positive by IgM capture ELISA. These results suggest that the IgM capture ELISA may be slightly more sensitive than the WB. In fact, the IgM capture ELISA has been shown by others to have better sensitivity than another assay, the 19S IgM FTA-ABS test (29). While the ELISA may have some practical advantages in terms of assay performance and objectivity, additional comparative studies are needed to determine whether it performs better than WB. We cannot comment on the specificity of the IgM capture ELISA because not all sera were analyzed by this method.

Since IgA antibodies, like IgM, do not cross the placenta, they are also potential markers of congenital infection. Recently, the use of IgA WB for the diagnosis of congenital human immunodeficiency virus infection yielded results superior to those obtained by IgM blotting $(14,21,31)$. The use of IgA antibodies for detecting infants at risk for congenital syphilis was less promising than the use of IgM antibodies, however. We detected IgA antibodies in four of six $(67 \%)$ infants with symptomatic congenital syphilis. This finding is similar to that of Pedersen et al. (20), who demonstrated $70 \%$ sensitivity for an IgA ELISA among a group of 10 infants born to mothers with untreated syphilis. Two asymptomatic infants (no. 96 and 103) were also found to have positive IgA blots. Their mothers reported treatment for syphilis 5 months and 2 years before delivery, respectively. Thus, the significance of the reactive IgA blots is not clear, although reinfection or treatment failure cannot be ruled out. Further follow-up of these infants would be desirable but is not possible for our study. Like the IgM blots, all the IgA-positive blots reacted to the 47-kDa antigen. In addition, both higher- and lower-molecular-mass proteins were recognized by some of the samples. In general, however, the intensity of the bands was less than in IgM blots.

Comparative mother-infant IgG WBs were also performed for the symptomatic infants in this study. Our present finding that the reactivity of maternal and infant sera is very similar is not surprising. One would expect the presence of maternally acquired IgG in the infant's serum to result in similar WB patterns. We, however, found two sera from congenitally infected, symptomatic infants that contained antibodies to a spirochetal protein not recognized, or only weakly recognized, by their respective maternal sera. This protein may correspond to the 83-kDa protein described by Dobson et al. (7). However, our findings suggest that the usefulness of the reaction is limited because of its infrequent occurrence. We did not notice differences in IgG WB results for asymptomatic infant-mother pairs.

A much more consistent finding was IgM and IgA reactivity to the $47-\mathrm{kDa}$ antigen by sera from infants at risk for congenital syphilis. As in other studies (7, 17, 23-25), the majority of our IgM-reactive sera (seven of eight) demonstrated this reactivity. We found much less reactivity to other proteins, such as those of 45,17 , and $15 \mathrm{kDa}$. We may be able to improve the reactivity to other proteins, however, by using Triton X-114 phase partitioning to enrich for lipoproteins, as described by Sanchez et al. (24). The infant sample (no. 76) with IgM reactivity to a protein of approximately $93 \mathrm{kDa}$ (and not to the $47-\mathrm{kDa}$ protein) was an unexpected finding. While the maternal serum reacted to this protein, it also recognized $47-$ and $45-\mathrm{kDa}$ proteins with similar intensity. The $47-$ and $45-\mathrm{kDa}$ bands, however, were not seen with the infant's sample. Thus, we do not believe that the reactivity of the infant's sample was due to contamination by maternal blood. 
Potential drawbacks to IgM WB include interference from RF and high concentrations of $T$. pallidum-specific IgG antibodies, which may compete with IgM and IgA antibodies. These factors can result in false-positive and false-negative IgM serological tests, respectively. It is important to study the effect of these potential interferences in IgM WB for congenital syphilis because many infected infants have RF $(18,22)$. We detected RF in four of eight infant sera tested. In one case (infant 103), removal of IgG by protein $G$ absorption changed the blot result to negative (reactivity to the $47-\mathrm{kDa}$ antigen was abolished). We do not believe that this represents aberrant removal of $T$. pallidum-specific IgM from the sample because decreased IgM reactivity was not noted to occur in other samples after absorption (even in RF-negative samples). In addition, several sera demonstrated increased $\operatorname{IgM}$ and $\operatorname{IgA}$ band intensities after absorption of IgG, suggesting competition between the two isotypes of antibody and not removal of IgM or IgA antibodies. Since these interfering substances can cause problems in syphilis IgM blotting assays, we believe that it is prudent to absorb IgG from the patient's serum sample before testing. Others have noted alterations in WB profiles after absorption of IgG from sera (17).

Our findings confirm those of previous studies demonstrating the utility of IgM WB in the diagnosis of congenital syphilis $(7,17,23,24-26,32)$. In addition, our data suggest that IgA antibodies detectable by WB can be used as additional markers of congenital syphilis. Further development of IgM and IgA serologic tests and DNA amplification techniques should greatly facilitate the diagnosis of congenital syphilis.

\section{ACKNOWLEDGMENTS}

We thank the serology laboratory at Carolinas Medical Center for performing VDRL analyses, Robert Baughn for providing positive control sera, Sandra Larsen for performing the IgM capture ELISAs, and Eloise Parrish for technical assistance in obtaining T. pallidum cells.

\section{REFERENCES}

1. Alpert, G., and S. A. Plotkin. 1986. A practical guide to the diagnosis of congenital infection in the newborn infant. Pediatr. Clin. N. Am. 33:465-479.

2. Berry, M. C., and A. S. Dajani. 1992. Resurgence of congenital syphilis. Infect. Dis. Clin. N. Am. 6:19-29.

3. Bromberg, K., S. Rawstron, and G. Tannis. 1993. Diagnosis of congenital syphilis by combining Treponema pallidum-specific IgM detection with immunofluorescent antigen detection for $T$. pallidum. J. Infect. Dis. 168:238-242.

4. Bromberg, K., S. Rawstron, G. Tannis, A. Hadero, and S. Jenkins. 1990. Diagnosis of congenital syphilis (CS) by Ortho IgG and IgM T. pallidum (TP) enzyme immunoassay, abstr. C-109, p. 362. Abstr. 90th Annu. Meet. Am. Soc. Microbiol. 1990.

5. Centers for Disease Control. 1988. Guidelines for the prevention and control of congenital syphilis. Morbid. Mortal. Weekly Rep. 37(Suppl. 1):1.

6. Centers for Disease Control. 1991. Primary and secondary syphilis-United States, 1981-1990. Morbid. Mortal. Weekly Rep. 40:314.

7. Dobson, S. R. M., L. H. Taber, and R. E. Baughn. 1988. Recognition of $T$. pallidum antigens by IgM and IgG antibodies in congenitally infected newborns and their mothers. J. Infect. Dis. 157:903-910.

8. Dul, M. J. 1990. Evaluation of an enzyme immunoassay for the detection of IgM and IgG antibodies specific for Treponema pallidum, abstr. C-112, p. 362. Abstr. 90th Annu. Meet. Am. Soc. Microbiol. 1990.

9. Grimprel, E., P. J. Sanchez, G. D. Wendel, J. M. Burstain, G. H. McCracken, Jr., J. D. Radolf, and M. V. Norgard. 1991. Use of polymerase chain reaction and rabbit infectivity testing to detect Treponema pallidum in amniotic fluid, fetal and neonatal sera, and cerebrospinal fluid. J. Clin. Microbiol. 29:1711-1718.

10. Grodzicki, R. L., and A. C. Steere. 1988. Comparison of immunoblotting and indirect enzyme-linked immunosorbent assay using different antigen preparations for diagnosing early Lyme disease. J. Infect. Dis. 157:790-797.

11. Ikeda, M. K., and H. B. Jensen. 1990. Medical progress-evaluation and treatment of congenital syphilis. J. Pediatr. 117:843-852.

12. Kaufman, R. E., D. C. Olansky, and P. J. Weisner. 1974. The FTA-ABS (IgM) test for neonatal congenital syphilis: a critical review. J. Am. Vener. Dis. Assoc. 1:79-84.

13. Laemmli, U. K. 1970. Cleavage of structural proteins during the assembly of the head of bacteriophage T4. Nature (London) 227:680-685.

14. Landesman, S., B. Weiblen, J. Mendez, A. Willoughby, J. J. Goedert, A. Rubinstein, J. Minkoff, and G. Moroso. 1991. Clinical utility of HIV-IgA immunoblot in the early diagnosis of perinatal HIV infections. J. Am. Med. Assoc. 266:3443-3446.

15. Lewis, L. L. 1992. Congenital syphilis-serologic diagnosis in the young infant. Infect. Dis. Clin. N. Am. 6:31-39.

16. Lewis, L. L., L. H. Taber, and R. E. Baughn. 1989. The Venereal Disease Research Laboratory (VDRL)-IgM ELISA as a screening test for congenital syphilis. Program Abstr. 29th Intersci. Conf. Antimicrob. Agents Chemother., abstr. 945.

17. Lewis, L. L., L. H. Taber, and R. E. Baughn. 1990. Evaluation of immunoglobulin $\mathrm{M}$ Western blot analysis in the diagnosis of congenital syphilis. J. Clin. Microbiol. 28:296-302.

18. Meyer, M. P., and D. W. Beatty. 1991. IgM rheumatoid factor in congenital syphilis: association with clinical and laboratory findings. Clin. Exp. Immunol. 86:43-48.

19. Muller, F. 1986. Specific immunoglobulin $M$ and $G$ antibodies in the rapid diagnosis of human treponemal infections. Diagn. Immunol. 4:1-9.

20. Pedersen, N. S., J. P. Sheller, A. V. Ratnam, and S. K. Hira. 1989. Enzyme-linked immunosorbent assays for detection of immunoglobulin $M$ to nontreponemal and treponemal antigens for the diagnosis of congenital syphilis. J. Clin. Microbiol. 27:1835-1840.

21. Quinn, T., R. L. Kline, N. Halsey, N. Hutton, A. Raff, A. Butz, R. Boulos, and J. F. Modlin. 1991. Early diagnosis of perinatal HIV infection by detection of viral-specific IgA antibodies. J. Am. Med. Assoc. 266:3439-3442.

22. Reimer, C. B., C. M. Black, D. J. Phillips, L. C. Logan, E. F. Hunter, B. J. Pender, and B. E. McGrew. 1975. The specificity of fetal IgM. Antibody or anti-antibody? Ann. N.Y. Acad. Sci. 254:77-93.

23. Sanchez, P. J., G. H. McCracken, Jr., G. D. Wendel, K. Olsen, N. Threlkeld, and M. V. Norgard. 1989. Molecular analysis of the fetal IgM response to Treponema pallidum antigens: implications for improved serodiagnosis of congenital syphilis. J. Infect. Dis. 159:508-517.

24. Sanchez, P. J., G. D. Wendel, Jr., E. Grimprel, M. Goldberg, M. Hall, O. Arencibia-Mireles, J. D. Radolf, and M. V. Norgard. 1993. Evaluation of molecular methodologies and rabbit infectivity testing for the diagnosis of congenital syphilis and neonatal central nervous system invasion by Treponema pallidum. J. Infect. Dis. 167:148-157.

25. Sanchez, P. J., G. D. Wendel, G. H. McCracken, Jr., and M. V. Norgard. 1989. Fetal IgM response to Treponema pallidum antigens in asymptomatic infants with suspected congenital syphilis, abstr. 1669. Pediatr. Res. 25:281A.

26. Sanchez, P. J., G. D. Wendel, and M. V. Norgard. 1992. IgM antibody to Treponema pallidum in cerebrospinal fluid of infants with congenital syphilis. Am. J. Dis. Child. 146:1171-1175.

27. Schwartz, J. S., P. G. Dans, and B. P. Kinosian. 1988. Human immunodeficiency virus test evaluation, performance, and use: proposals to make good tests better. J. Am. Med. Assoc. 259:25742579.

28. Senna, J., E. Sargent, J. Herbert, P. Simon, and C. Peter. 1990. Comparison of Ortho Diagnostic Systems, Inc., Syphilis-G and Syphilis-M antibody ELISA test with conventional serologic methods for diagnosis of congenital syphilis, abstr. C-108, p. 362. Abstr. 90th Annu. Meet. Am. Soc. Microbiol. 1990.

29. Stoll, B. J., F. K. Lee, S. Larsen, E. Hale, D. Schwartz, R. J. Rice, R. Ashby, R. Holmes, and A. J. Nahmias. 1993. Clinical and 
serologic evaluation of neonates for congenital syphilis: a continuing diagnostic dilemma. J. Infect. Dis. 167:1093-1099.

30. Towbin, H., T. Staehelin, and J. Gordon. 1979. Electrophoretic transfer of proteins from polyacrylamide gels to nitrocellulose sheets: procedure and some applications. Proc. Natl. Acad. Sci. USA 76:4350-4354.

31. Weiblen, B. J., F. K. Lee, E. R. Cooper, S. H. Landesman, K.
McIntosh, J. S. Harris, S. Nesheim, H. Mendez, S. I. Pelton, A. J. Nahmias, and R. Hoff. 1990. Early diagnosis of HIV infection in infants by detection of IgA antibodies. Lancet 335:988-990.

32. Wendel, G. D., Jr., P. J. Sanchez, M. T. Peters, T. W. Harstad, L. L. Potter, and M. V. Norgard. 1991. Identification of Treponema pallidum in amniotic fluid and fetal blood from pregnancies complicated by congenital syphilis. Obstet. Gynecol. 78:890-895. 